\title{
Curvature instability of a curved Batchelor vortex
}

\author{
Francisco J. Blanco-Rodríguez and Stéphane Le Dizès ${ }^{\dagger}$ \\ Aix Marseille Université, CNRS, Centrale Marseille, IRPHE, Marseille, France
}

(Received 10 January 2017)

In this paper, we analyse the curvature instability of a curved Batchelor vortex. We consider this short-wavelength instability when the radius of curvature of the vortex centerline is large compared to the vortex core size. In this limit, the curvature instability can be interpreted as a resonant phenomenon. It results from the resonant coupling of two Kelvin modes of the underlying Batchelor vortex with the dipolar correction induced by curvature. The condition of resonance of the two modes is analysed in detail as a function of the axial jet strength of the Batchelor vortex. Contrarily to the Rankine vortex, only a few configurations involving $m=0$ and $m=1$ modes are found to become the most unstable. The growth rate of the resonant configurations is systematically computed and used to determine the characteristics of the most unstable mode as a function of the curvature ratio, the Reynolds number, and the axial flow parameter. The competition of the curvature instability with another short-wavelength instability, which was considered in a companion paper [Blanco-Rodríguez \& Le Dizès, Elliptic instability of a curved Batchelor vortex, J. Fluid Mech. 804, 224-247 (2016)], is analysed for a vortex ring. A numerical error found in this paper which affects the relative strength of the elliptic instability is also corrected. We show that the curvature instability becomes the dominant instability in large rings as soon as axial flow is present (vortex ring with swirl).

\section{Introduction}

Vortices are ubiquitous in nature. They are subject to various instabilities induced by the interaction with their surroundings. In this work, we analyse the so-called curvature instability which is a short-wavelength instability induced by the local curvature of the vortex. We provide theoretical predictions for a curved vortex when the underlying vortex structure is a Batchelor vortex (Gaussian axial velocity and axial vorticity). This work is the follow-up of Blanco-Rodríguez \& Le Dizès (2016), hereafter BRLD16, where another short-wavelength instability, the elliptic instability, was analysed using the same theoretical framework.

These two instabilities are different from the long-wavelength instabilities which occur in vortex pairs (Crow 1970) and helical vortices (Widnall 1972; Quaranta et al. 2015). Their characteristics strongly depend on the internal vortex structure and their wavelength is of the order of the vortex core size. When the vortex is weakly deformed, both instabilities can be understood as a phenomenon of resonance between two (Kelvin) modes of the underlying vortex and a vortex correction. For the elliptic instability, the resonance occurs with a quadripolar correction generated by the background strain field (Moore \& Saffman 1975), while for the curvature instability, it is associated with a dipolar correction created by the vortex curvature (Fukumoto \& Hattori 2005). Numerous works have concerned the elliptic instability in the context of straight vortices (Tsai \& 
Widnall 1976; Eloy \& Le Dizès 1999; Fabre \& Jacquin 2004a; Lacaze et al. 2007). The specific case of the curved Batchelor vortex has been analysed in BRLD16. Contrarily to the elliptic instability, the curvature instability has only been considered for vortices with uniform vorticity (Fukumoto \& Hattori 2005; Hattori \& Fukumoto 2014).

Both elliptic and curvature instabilities have also been analysed using the local Lagrangian method popularized by Lifschitz \& Hameiri (1991) [see Bayly (1986); Waleffe (1990) for the elliptic instability, Hattori \& Fukumoto (2003, 2009, 2012) for the curvature instability]. This method can be used to treat strongly deformed vortices but it provides a local information on a given streamline only. When the vortex is uniform, as the Rankine vortex, the local instability growth rate is also uniform. In that case, a connection can be made between the local results and the global results obtained by analyzing the mode resonances (Waleffe 1990; Eloy \& Le Dizès 2001; Fukumoto 2003; Hattori \& Fukumoto 2010, 2014). Le Dizès \& Laporte (2002) used the local prediction at the vortex centre to estimate the global growth rate of the elliptic instability in a non-uniform vortex. Although a good agreement was demonstrated for the Lamb-Oseen vortex, no such link is expected in general.

The goal of the present work is to obtain global estimates for the curvature instability using the framework of Moore \& Saffman (1975) for the Batchelor vortex. Such an analysis was performed by Hattori \& Fukumoto (2014) for a Rankine vortex. The passage from the Rankine vortex to the Batchelor vortex will turn out not to be trivial. The main reason comes from the different properties of the Kelvin modes in both vortices. In smooth vortices, Kelvin modes are affected by the presence of critical layers (Le Dizès 2004) which introduce singularities and damping (Sipp \& Jacquin 2003; Fabre et al. 2006). These singularities have to be monitored and avoided in the complex plane to be able to obtain the properties of the Kelvin modes from the inviscid equations as shown in Lacaze et al. (2007). In the present work, we shall also use the asymptotic theory of Le Dizès \& Lacaze (2005) to obtain an approximation of the Kelvin mode dispersion relation and analyse the condition of resonance.

The dipolar correction responsible of the curvature instability is also obtained by an asymptotic theory in the limit of small vortex core size (Callegari \& Ting 1978). This correction appears as a first order correction to the Batchelor vortex. The detail of the derivation can be found in Blanco-Rodríguez et al. (2015). As for the elliptic instability, the coupling terms, as well as weak detuning and viscous effects are computed using an orthogonality condition. The final result is an expression for the growth rate of a given resonant configuration close to the condition of resonance. Each resonant configuration provides a growth rate expression. We shall consider up to 50 resonant configurations to extract the most unstable one. This will allows us to obtain the curvature instability diagram as a function of the curvature ratio and the Reynolds number.

The paper is organized as follows. In $\S 2$, the base flow and perturbation equations are provided. In $\S 3$, the analysis leading the growth rate expression of a resonant configuration is presented. The results for the Batchelor vortex are obtained in $\S 4$. We first provide the characteristics of the resonant modes, then the stability diagrams for the Batchelor vortex for a few values of the axial flow parameter. Section $\S 5$ provides an application of the results to a vortex ring with and without swirl (axial flow). In that section, we analyse the competition of the curvature with the elliptic instability using the results of BRLD16. A numerical error affecting the strength of the elliptic instability has been found in this paper. It is corrected in a corrigendum which is presented in appendix D. The last section $\S 6$ gives a brief summary of the main results of the paper. 


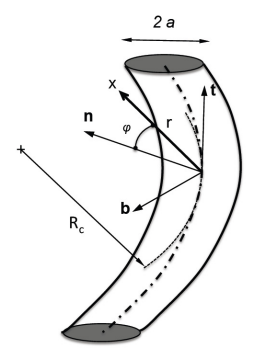

Figure 1. Sketch of the vortex structure and definition of the local Frenet frame (adapted from BRLD16).

\section{Problem formulation}

\subsection{Base flow}

The first description of the base flow was provided by Callegari \& Ting (1978). Here, as in BRLD16, we mainly follow the presentation given in Blanco-Rodríguez et al. (2015). The vortex is considered in the local Frenet frame $(\mathbf{t}, \mathbf{n}, \mathbf{b})$ attached to the vortex centerline and moving with the structure. We assume that the vortex is concentrated (i.e. thin), which means that its core size $a$ is small compared to the local curvature radius $R_{c}$ of the vortex centerline and the shortest separation distance $\delta$ to other vortex structures. For simplicity, we consider a single small parameter $\varepsilon=a / R_{c}$, and assume that $\delta=O\left(R_{c}\right)$. The internal vortex dynamics is described using the "cylindrical" coordinate system $(r, \varphi, s)$ constructed from the Frenet frame (see Fig. 1).

The velocity-pressure field of the base flow is expanded in power of $\varepsilon$ as $\mathbf{U}=\mathbf{U}_{\mathbf{0}}+$ $\varepsilon \mathbf{U}_{1}+\cdots$. The leading order contribution is the prescribed Batchelor vortex of velocity field $\mathbf{U}_{0}=\left(0, V^{(0)}(r), W^{(0)}(r), P^{(0)}(r)\right)$ with

$$
V^{(0)}=\frac{1-\mathrm{e}^{-r^{2}}}{r}, \quad W^{(0)}=W_{0} \mathrm{e}^{-r^{2}} .
$$

As in BRLD16, spatial and time scales have been non-dimensionalized using the core size $a$ and the maximum angular velocity of the vortex $\Omega_{\max }^{(0)}=\Gamma /\left(2 \pi a^{2}\right), \Gamma$ being the vortex circulation. The axial flow parameter $W_{0}$ is defined as the ratio

$$
W_{0}=\frac{W_{\max }^{(0)}}{\Omega_{\max }^{(0)} a} .
$$

We assume that $W_{0} \leqslant 0.5$ such that the vortex remains unaffected by the inviscid swirling jet instability (Mayer \& Powell 1992). We also implicitly assume that the weak viscous instabilities occurring for small values of $W_{0}$ (Fabre \& Jacquin 2004b; Le Dizès \& Fabre 2007) remain negligible in the parameter regime that is considered. In the following, we shall also use the expression of the angular velocity $\Omega^{(0)}(r)$ and vorticity $\zeta^{(0)}(r)$ :

$$
\Omega^{(0)}(r)=\frac{1-\mathrm{e}^{-r^{2}}}{r^{2}}, \quad \zeta^{(0)}(r)=2 \mathrm{e}^{-r^{2}} .
$$

As explained by Blanco-Rodríguez et al. (2015), the first order correction is a dipolar 
field which can be written as

$$
\mathbf{U}_{1} \sim \varepsilon \operatorname{Re}\left(\mathbf{U}^{(1)} \mathrm{e}^{\mathrm{i} \varphi}\right)=\frac{\varepsilon}{2}\left(\begin{array}{c}
\mathrm{i} U^{(1)}(r) \\
V^{(1)}(r) \\
W^{(1)}(r) \\
P^{(1)}(r)
\end{array}\right) \mathrm{e}^{\mathrm{i} \varphi}+\text { c.c. }
$$

where expressions for $U^{(1)}, V^{(1)}, W^{(1)}$ and $P^{(1)}$ are provided in appendix A. It is worth emphasizing that these expressions only depend on the local characteristics of the vortex at leading order. In particular, they do not depend on the local torsion. For helices, torsion as well as the Coriolis effects associated with the change of frame appear at second order (Hattori \& Fukumoto 2009). The above expression then describes the internal structure of both helices and rings up to the order $\varepsilon$. This contrasts with the quadripolar correction responsible of the elliptic instability which appears at second order. This quadripolar correction varies according to the global vortex geometry and is different for rings and helices even if they have the same local curvature.

\subsection{Perturbation equations}

The perturbations equations are obtained by linearizing the governing equations around the base flow $\mathbf{U}=\mathbf{U}_{0}+\varepsilon \mathbf{U}_{\mathbf{1}}+\cdots$. As shown in BRLD16, if the perturbation velocitypressure field is written as $\mathbf{u}=(-\mathrm{i} u, v, w, p)$ we obtain up to $\mathrm{o}(\varepsilon)$ terms a system of the form :

$$
\left(\mathrm{i} \partial_{t} \mathbf{I}+\mathrm{i} \partial_{s} \mathbf{P}+\mathbf{M}\right) \mathbf{u}=\varepsilon\left(\mathrm{e}^{\mathrm{i} \varphi} \mathbf{N}_{+}^{(1)}+\mathrm{e}^{-\mathrm{i} \varphi} \mathbf{N}_{-}^{(1)}\right) \mathbf{u}+\frac{\mathrm{i}}{R e} \mathbf{V u}
$$

where the operators $\mathbf{I}, \mathbf{P}, \mathbf{M}=\mathbf{M}\left(-\mathrm{i} \partial_{\varphi}\right), \mathbf{N}_{ \pm}^{(1)}=\mathbf{N}_{ \pm}^{(1)}\left(-\mathrm{i} \partial_{\varphi},-\mathrm{i} \partial_{s}\right), \mathbf{V}=\mathbf{V}\left(-\mathrm{i} \partial_{\varphi},-\mathrm{i} \partial_{s}\right)$ are defined in Appendix B.

The left-hand side corresponds to the inviscid perturbation equations of the undeformed Batchelor vortex. The first term on the right-hand side is responsible of the curvature instability, while the second term accounts for the viscous effects on the perturbations. By introducing viscous effects in this equation, we implicitly assume that the Reynolds number

$$
R e=\frac{\Omega_{\max }^{(0)} a^{2}}{\nu}=\frac{\Gamma}{2 \pi \nu},
$$

with $\nu$ the kinematic viscosity, is of order $1 / \varepsilon$.

\section{Instability description}

\subsection{Curvature instability mechanism}

The mechanism of the curvature instability is similar to that of the elliptic instability. The instability results from a resonant coupling of two Kelvin modes of the undeformed axisymmetric vortex with non-axisymmetric corrections. Two Kelvin modes of characteristics $\left(\omega_{A}, k_{A}, m_{A}\right)$ and $\left(\omega_{B}, k_{B}, m_{B}\right)$ are resonantly coupled via the dipolar correction if they satisfy the condition of resonance (assuming $m_{A}<m_{B}$ )

$$
\omega_{A}=\omega_{B}, k_{A}=k_{B}, m_{A}=m_{B}-1
$$

Fukumoto (2003) further demonstrated that the coupling is destabilizing only if the energy of the modes is opposite or if the frequency vanishes. It leads to a growth of the Kelvin mode combination with a maximum growth rate scaling as $\varepsilon$. 
3.2. Formal derivation of the growth rate formula

For each resonant configuration, a growth rate expression can be obtained from an orthogonality condition as we did for the elliptic instability (see BRLD16). We consider a combination of two Kelvin modes of azimuthal wavenumber $m_{A}$ and $m_{B}=m_{A}+1$ close to the their condition of resonance (3.1):

$$
\mathbf{u}=\left(A \tilde{\mathbf{u}}_{A}(r) \mathrm{e}^{\mathrm{i} m_{A} \varphi}+B \tilde{\mathbf{u}}_{B}(r) \mathrm{e}^{\mathrm{i} m_{B} \varphi}\right) \mathrm{e}^{\mathrm{i} k s-\mathrm{i} \omega t}
$$

where $k$ is close to $k_{A}=k_{B}=k_{c}$, and $\omega$ close to $\omega_{A}=\omega_{c}$ and $\omega_{B}=\omega_{c}+\mathrm{i} \operatorname{Im}\left(\omega_{B}\right)$. We assume that the resonance is not perfect. The mode B will exhibit a weak critical layer damping given by $\operatorname{Im}\left(\omega_{B}\right)$ (imaginary part of $\left.\omega_{B}\right)$. The functions $\tilde{\mathbf{u}}_{A}(r)$ and $\tilde{\mathbf{u}}_{B}(r)$ are the eigenfunctions of the Kelvin modes which satisfy

$$
\begin{aligned}
& \left(\omega_{A} \mathbf{I}-k_{A} \mathbf{P}+\mathbf{M}\left(m_{A}\right)\right) \tilde{\mathbf{u}}_{A}=0, \\
& \left(\omega_{B} \mathbf{I}-k_{B} \mathbf{P}+\mathbf{M}\left(m_{B}\right)\right) \tilde{\mathbf{u}}_{B}=0,
\end{aligned}
$$

with a prescribed normalisation:

$$
\tilde{p}_{A} \underset{r \rightarrow 0}{\sim} r^{\left|m_{A}\right|}, \quad \tilde{p}_{B} \underset{r \rightarrow 0}{\sim} r^{\left|m_{B}\right|} .
$$

If we plug (3.2) in (2.5), we obtain for the components proportional to $e^{\mathrm{i} m_{A} \varphi}$ and $e^{\mathrm{i} m_{B} \varphi}$ :

$$
\begin{aligned}
& A\left(\omega \mathbf{I}-k \mathbf{P}+\mathbf{M}\left(m_{A}\right)-\frac{\mathrm{i}}{R e} \mathbf{V}\left(m_{A}, k\right)\right) \tilde{\mathbf{u}}_{A}=B \varepsilon \mathbf{N}_{-}^{(1)}\left(m_{B}, k\right) \tilde{\mathbf{u}}_{B} \\
& B\left(\omega \mathbf{I}-k \mathbf{P}+\mathbf{M}\left(m_{B}\right)-\frac{\mathrm{i}}{R e} \mathbf{V}\left(m_{B}, k\right)\right) \tilde{\mathbf{u}}_{B}=A \varepsilon \mathbf{N}_{+}^{(1)}\left(m_{A}, k\right) \tilde{\mathbf{u}}_{A}
\end{aligned}
$$

Relations between the amplitudes $A$ and $B$ are obtained by projecting these equations on the subspace of the adjoint Kelvin modes. We define the adjoint eigenfunctions $\tilde{\mathbf{u}}_{A}^{\dagger}$ and $\tilde{\mathbf{u}}_{B}^{\dagger}$ of the Kelvin modes as the solutions to the adjoint equations of (3.3)-(3.4) with respect to the scalar product

$$
<\mathbf{u}_{1}, \mathbf{u}_{2}>=\int_{0}^{\infty} \mathbf{u}_{1}^{\star} \cdot \mathbf{u}_{2} r d r=\int_{0}^{\infty}\left(u_{1}^{\star} u_{2}+v_{1}^{\star} v_{2}+w_{1}^{\star} w_{2}+p_{1}^{\star} p_{2}\right) r d r .
$$

We then obtain

$$
\begin{gathered}
\left(\omega-\omega_{c}-Q_{A}\left(k-k_{c}\right)-\mathrm{i} \frac{V_{A}}{R e}\right) A=\varepsilon C_{A B} B, \\
\left(\omega-\omega_{c}-\mathrm{i} \operatorname{Im}\left(\omega_{B}\right)-Q_{B}\left(k-k_{c}\right)-\mathrm{i} \frac{V_{B}}{R e}\right) B=\varepsilon C_{B A} A,
\end{gathered}
$$

where the coefficients of these equations are given by

$$
\begin{aligned}
& Q_{A}=\frac{<\tilde{\mathbf{u}}_{A}^{\dagger}, \mathbf{P} \tilde{\mathbf{u}}_{A}>}{<\tilde{\mathbf{u}}_{A}^{\dagger}, \mathbf{I} \tilde{\mathbf{u}}_{A}>}, \quad Q_{B}=\frac{<\tilde{\mathbf{u}}_{B}^{\dagger}, \mathbf{P} \tilde{\mathbf{u}}_{B}>}{<\tilde{\mathbf{u}}_{B}^{\dagger}, \mathbf{I} \tilde{\mathbf{u}}_{B}>} \\
& V_{A}=\frac{<\tilde{\mathbf{u}}_{A}^{\dagger}, \mathbf{V} \tilde{\mathbf{u}}_{A}>}{<\tilde{\mathbf{u}}_{A}^{\dagger}, \mathbf{I} \tilde{\mathbf{u}}_{A}>}, \quad V_{B}=\frac{<\tilde{\mathbf{u}}_{B}^{\dagger}, \mathbf{V} \tilde{\mathbf{u}}_{B}>}{<\tilde{\mathbf{u}}_{B}^{\dagger}, \mathbf{I} \tilde{\mathbf{u}}_{B}>}, \\
& C_{A B}=\frac{<\tilde{\mathbf{u}}_{A}^{\dagger}, \mathbf{N}_{+}^{(1)}\left(m_{B}, k_{B}\right) \tilde{\mathbf{u}}_{B}>}{<\tilde{\mathbf{u}}_{A}^{\dagger}, \mathbf{I} \tilde{\mathbf{u}}_{A}>}, C_{B A}=\frac{<\tilde{\mathbf{u}}_{B}^{\dagger}, \mathbf{N}_{-}^{(1)}\left(m_{A}, k_{A}\right) \tilde{\mathbf{u}}_{A}>}{<\tilde{\mathbf{u}}_{B}^{\dagger}, \mathbf{I} \tilde{\mathbf{u}}_{B}>} .
\end{aligned}
$$


The formula for the complex frequency $\omega$ is then finally given by

$$
\left(\omega-\omega_{c}-\mathrm{i} \operatorname{Im}\left(\omega_{B}\right)-Q_{B}\left(k-k_{c}\right)-\mathrm{i} \frac{V_{B}}{R e}\right)\left(\omega-\omega_{c}-Q_{A}\left(k-k_{c}\right)-\mathrm{i} \frac{V_{A}}{R e}\right)=-\varepsilon^{2} N^{2},
$$

with

$$
N=\sqrt{-C_{A B} C_{B A}} .
$$

The right-hand side of (3.14) represents the coupling terms responsible of the curvature instability. The left-hand side of (3.14) gives the dispersion relation of each Kelvin mode close to the resonant point. It is important to mention that none of the coefficients $Q_{A}$, $Q_{B}, V_{A}, V_{B}$ and $N$ depends on the normalization chosen for the Kelvin modes.

\section{Instability results for the Batchelor vortex profile}

\subsection{Resonant Kelvin modes}

The main difficulty of the analysis is to determine the Kelvin modes that satisfy the condition of resonance (3.1). A similar problem was already addressed in Lacaze et al. (2007). The Kelvin modes are here defined from the inviscid equations. Two kinds of Kelvin modes are found to exist: the regular and neutral Kelvin modes which can easily be obtained by integrating the inviscid perturbation equations in the physical domain and the singular and damped Kelvin modes which require a particular monitoring of the singularities of the perturbation equations in the complex plane. We shall see below that the condition of resonance always involves a singular mode.

The singularities of the inviscid perturbation equations are the critical points $r_{c}$ where $\omega-k W^{(0)}\left(r_{c}\right)-m \Omega^{(0)}\left(r_{c}\right)=0$. When $\operatorname{Im}(\omega)>0$, these singularities are in the complex plane, and do not affect the solution in the physical domain (real $r$ ). However, one of such critical points may cross the real axis when $\operatorname{Im}(\omega)$ becomes negative. As explained in Le Dizès (2004), the inviscid equations must in that case be integrated on a contour in the complex $r$ plane that avoids the critical point from below (resp. above) if the critical point has moved in the lower (resp. upper) part of the complex plane. On such a contour, the solution remains regular and fully prescribed by the inviscid equations. On the real axis, the inviscid solution is however not regular anymore. As illustrated in Fabre et al. (2006), it no longer represents the vanishing viscosity limit of a viscous solution in a large interval of the physical domain. The Kelvin mode forms by the contour deformation technique is damped and singular. The inviscid frequency of the mode then possesses a negative imaginary part, which corresponds to what we call the critical layer damping rate. By definition, the critical layer damping rate is independent of viscosity.

A mode cannot be involved in a resonance if it is too much damped. In the asymptotic framework, the growth rate associated with the resonance is expected to be $O(\varepsilon)$, so the damping rate of the modes should a priori be asymptotically small of order $\varepsilon$. However, in practice, we shall consider values of $\varepsilon$ up to 0.2 , and the maximum growth rate will turn out to be around $0.05 \varepsilon$. We shall then discard all the modes with a damping rate whose absolute value exceeds 0.01 .

\subsubsection{Predictions from the WKBJ analysis}

Le Dizès \& Lacaze (2005) showed that information on the spectrum of the Kelvin modes can be obtained using a large $k$ asymptotic analysis. They applied their theory to the Batchelor vortex and were able to categorize the neutral Kelvin modes in four different types: regular core modes, singular core modes, regular ring modes, singular ring modes. For each $m$, they provided the region of existence of each type of mode in a $\left(k W_{0}, \omega\right)$ 


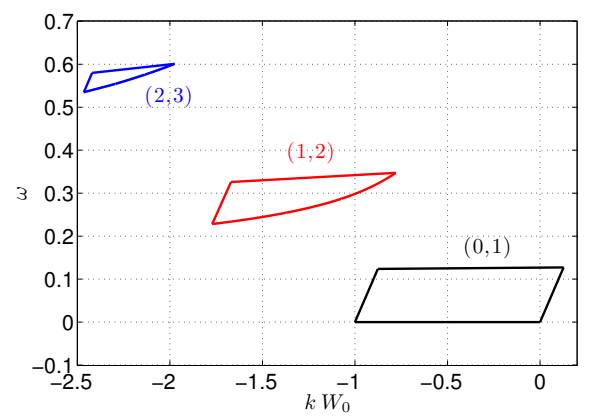

Figure 2. Prediction from the WKBJ analysis of the domains of parameters in the $\left(k W_{0}, \omega\right)$ plane where resonance between two Kelvin modes $\left(m_{A}, m_{A}+1\right)$ is possible. Only positive frequencies are considered. A symmetrical plot is obtained for negative frequencies.

plane. The energy of the waves can also be deduced from the asymptotic expression of the dispersion relation as shown in Le Dizès (2008). It is immediately found that regular core modes and regular ring modes are always of negative energy, while singular modes have positive energy.

The condition of resonance can then easily be analysed. One just needs to superimpose the domains of existence of each pair of modes of azimuthal wavenumbers $m$ and $m+1$, to find the regions of possible resonance. The final result is summarized in Fig. 2. For positive frequencies, only three different regions are obtained corresponding to $\left(m_{A}, m_{B}\right)=(0,1)$, $(1,2)$ and $(2,3)$ (Negative frequencies are obtained by symmetry changing $m \rightarrow-m$ and $k \rightarrow-k)$. No intersection of the domains of existence of the modes $m$ and $m+1$ are obtained for $m$ larger than 2 . In each region of Fig. 2, we always find that the mode $A$ is a regular core mode of negative energy, while the mode $B$ is a singular core mode of positive energy. Each branch crossing is therefore expected to provide an instability.

As shown in Le Dizès \& Lacaze (2005), both types of core modes have an asymptotic dispersion relation of the form

$$
k \int_{0}^{r_{t}} \frac{\sqrt{\Delta(r)}}{\Phi(r)} d r=(|m|+2 l) \frac{\pi}{2} \quad l=0,1,2, \ldots
$$

where

$$
\begin{aligned}
& \Delta(r)=2 \Omega^{(0)}(r) \zeta^{(0)}(r)-\Phi^{2}(r), \\
& \Phi(r)=\omega-m \Omega^{(0)}(r)-k W^{(0)}(r),
\end{aligned}
$$

and $r_{t}$ is a turning point defined by $\Delta\left(r_{t}\right)=0$. The integer $l$ is a branch label which measures the number of oscillations of the mode in the vortex core. The larger $l$, the more oscillating is the mode.

Singular modes differ from regular modes by the presence of a critical point $r_{c}>$ $r_{t}$ where $\Phi\left(r_{c}\right)=0$ in their radial structure. In the WKBJ description, this critical point does not create any damping at leading order. However, it makes the eigenfunction singular. It will justify the use of a complex integration path in the numerical resolution of the mode.

\subsubsection{Numerical determination of the Kelvin modes}

The characteristics of the resonant modes are obtained by integrating numerically Eqs. (3.3)-(3.4). The numerical code is based on a Chebyshev spectral collocation method, essentially identical to that used in Fabre \& Jacquin (2004b). 


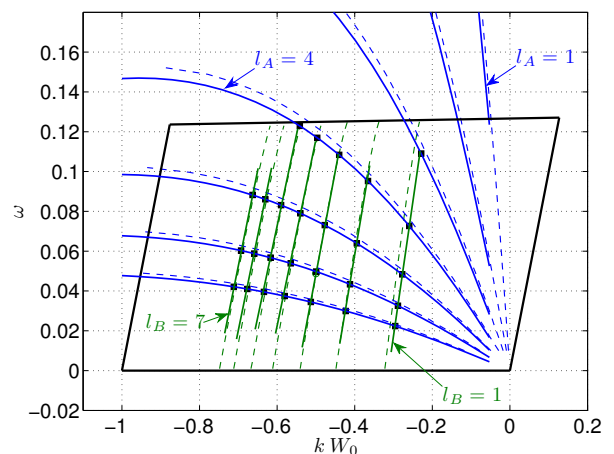

(a)

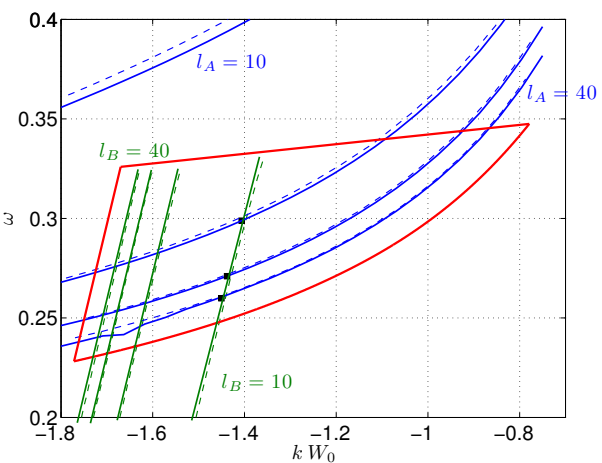

(b)

Figure 3. Analysis of the branch crossing for the Batchelor vortex at $W_{0}=0.2$. Plot of $\operatorname{Re}(\omega)$ versus $k W_{0}$ of the first branches of the Kelvin modes of azimuthal wavenumber $m_{A}$ (in blue) and $m_{B}=m_{A}+1$ (in green). The branch labels are also indicated. Solid lines: numerical results. Dashed lines: WKBJ predictions. The domains shown in figure 2 where branch crossings are expected have also been indicated. (a): $\left(m_{A}, m_{B}\right)=(0,1) ;(\mathrm{b}):\left(m_{A}, m_{B}\right)=(1,2)$.

The eigenvalue problem is solved in a Chebyshev domain $(-1,1)$ on $2(N+1)$ nodes which is mapped on a line in the complex- $r$ plane using the mapping

$$
r\left(x ; A_{c}, \theta_{c}\right)=A_{c} \tanh (x) \mathrm{e}^{\mathrm{i} \theta_{c}},
$$

where $A_{c}$ is a parameter close to 1 that controls the spreading of the collocation points, and $\theta_{c}$ is the small inclination angle of the path in the complex $r$ plane. We typically take $\theta_{c} \approx \pi / 10$ such that the critical point of the singular mode is avoided. As in Fabre \& Jacquin (2004b), we take advantages of the parity properties of the eigenfunctions by expressing for odd $m$ (resp. even $m$ ), $\tilde{w}$ and $\tilde{p}$ on odd polynomials (resp. even) and $\tilde{u}$ and $\tilde{v}$ on even polynomials (resp. odd). It leads to a discretized eigenvalue problem of order $4 N$, which is solved using a global eigenvalue method. We also use an Arnoldi algorithm in order to follow specific eigenvalues and easily find the condition of resonance. In most computations, the value $N=200$ was found to be adequate.

This collocation method was also used to determine the adjoint modes and compute the integrals that define the coefficients in the growth rate equation (3.14).

Typical results for the eigenvalues are shown in Fig. 3. In this figure, we compare the numerical results with the theoretical formula (4.1). The good agreement demonstrates the usefulness of the asymptotic approach to obtain valuable estimates for the condition of resonance.

\subsection{Stability diagram}

\subsubsection{Lamb-Oseen vortex}

In this section, we assume that there is no axial flow. The underlying vortex is then a Lamb-Oseen vortex. For this vortex, Kelvin mode properties have been documented in Le Dizès \& Lacaze (2005) and Fabre et al. (2006) for $m=0$, 1, 2, 3. It was shown that the singular core modes become strongly damped as soon as the critical layer singularity moves in the vortex core. This gives a constraint on the frequency of the mode $B$ which has to be small. As a consequence, we immediately see that the only modes $\left(m_{A}, m_{B}\right)$ that can possibly resonate are the modes $\left(m_{A}, m_{B}\right)=(0,1)$. Moreover, the constraint on the frequency implies that only large branch labels of the mode $m_{A}=0$ will be able to resonate with a weakly damped mode $m_{B}=1$. In figure 4 , we show the crossing of the 


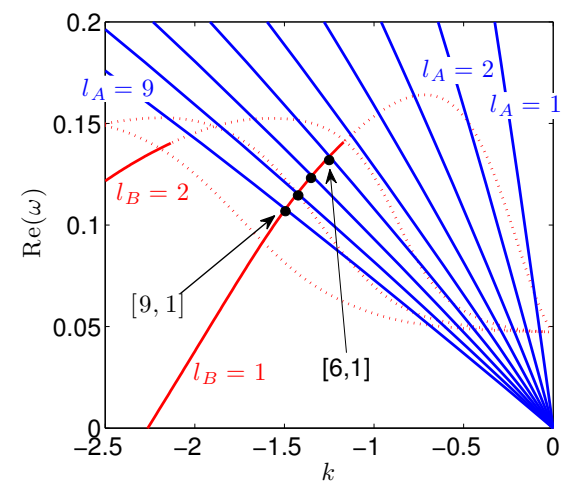

FIgURE 4. Frequency versus wavenumber of the Kelvin modes of the Lamb-Oseen vortex for $m_{A}=0$ (blue) and $m_{B}=1$ (red) in the frequency-wavenumber domain where resonance exists. The real part of the frequency is plotted in solid lines when $|\operatorname{Im}(\omega)|<0.01$ (neutral or weakly damped modes) and in dotted lines when $|\operatorname{Im}(\omega)|>0.01$ (strongly damped modes).

first $m_{A}=0$ and $m_{B}=1$ branches in the $(k, \omega)$ plane. Only the modes with a damping rate smaller (in absolute value) than 0.01 are in solid lines. We observe that the branch label of the $m_{A}=0$ modes must be 6 or larger to cross the first $m_{B}=1$ branch in the part where it is only weakly damped. The characteristics of these first resonance points are given in table 1. We also give in this table, the value of the coefficients of Eq. (3.14) at each resonant point. For each resonant configuration, we can then plot the growth rate $\operatorname{Im}(\omega)$ of the curvature instability as a function of the wavenumber $k$ for any $R e$ and $\varepsilon$. An example of such a plot is provided in Fig. 5. In this figure, we have plotted only the first four resonant configurations. Other configurations have been computed corresponding to labels $[2,6],[2,7]$, etc but their growth rates were found to be much weaker for $R e \leqslant 10^{5}$. The spatial structure of the most unstable resonant configurations are also been given in Fig. 5. We have plotted the vorticity contours for a particular phase which maximizes the relative amplitude of the Kelvin mode $m_{A}=0$. This mode is then clearly visible in each case. If we had chosen a phase such that $e^{\mathrm{i} k s-\mathrm{i} \omega t}=\mathrm{i}$, we would have seen the mode $m_{B}=1$ only.

We have systematically computed the maximum growth rate and obtained the most unstable mode characteristics for all $\varepsilon \leqslant 0.22$ and $R e \leqslant 10^{5}$. The result is displayed in Fig. 6 where the maximum growth rate is shown in the $(\varepsilon, R e)$ plane. The labels of the most unstable configurations are also shown in this plot. We can note that only 3 resonant configurations can become the most unstable corresponding to the crossing of the first branch of the Kelvin mode $m_{B}=1$ with the 7th to 9th branch of the Kelvin mode $m_{A}=0$. In particular, the resonant configuration [6,1] observed in Fig. 5 never becomes the most unstable configuration although this configuration possesses the largest coupling coefficient $N$ (see table 1 ). This is directly related to the property mentioned above: the critical layer damping rate $\operatorname{Im}\left(\omega_{B}\right)$ of the mode $m_{B}=1$ is too large.

Figure 6 provides the stability diagram of the Lamb-Oseen vortex with respect to the curvature instability. It is important to emphasize the large value of the Reynolds number needed for instability. Even for a value as large as $\varepsilon=0.2$, the critical Reynolds number for instability is $R e_{c} \approx 6000$. We shall see in the next section that axial flow will strongly decrease this value. 


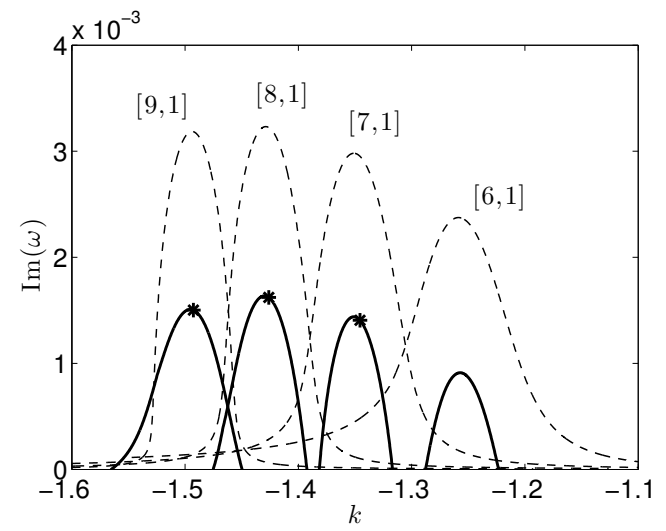

$[7,1]$

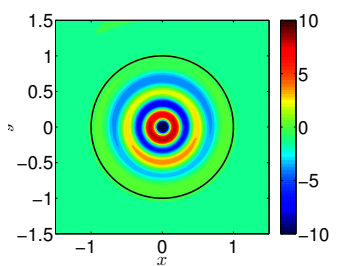

$[8,1]$

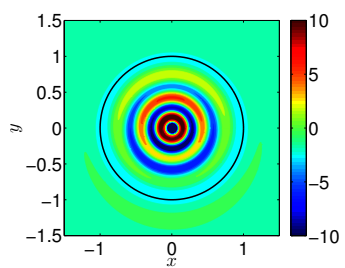

$[9,1]$

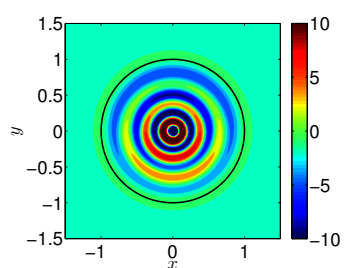

Figure 5. Top: Temporal growth rate of the curvature instability as a function of the axial wavenumber for the Lamb-Oseen vortex $\left(W_{0}=0\right)$ at $\varepsilon=0.1, R e=\infty$ (dashed line) and $R e=10^{5}$. The label $\left[l_{A}, l_{B}\right]$ corresponds to the branch indices of the resonant configuration. It means that the resonant configuration is formed of the $l_{A}$ th branch of the Kelvin mode $m_{A}=0$ and the $l_{B}$ th branch of the Kelvin mode $m_{B}=1$. Bottom: Vorticity contours in a $(x, y)$ cross section of modes $[7,1],[8,1]$, and $[9,1]$ for the parameters indicated by a star on the top graph (that is at $k=k_{c}$ ). The vorticity is defined by (3.2) at a time $t$ and location $s$ such that $e^{\mathrm{i} k s-\mathrm{i} \omega t}=1$ with $A=1$. The black circle indicates the vortex core radius.

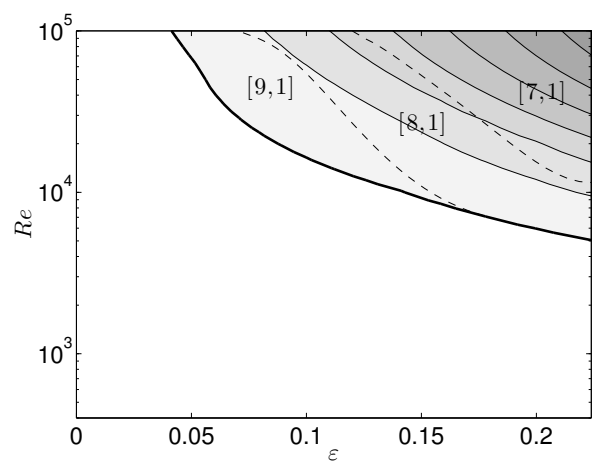

Figure 6. Maximum growth rate contour of the curvature instability in the $(\varepsilon, R e)$ plane for the Lamb-Oseen vortex $\left(W_{0}=0\right)$. Contours are every 0.001 . The thick solid line indicates the marginal stability curve. The dashed lines are the parameter boundaries where the most unstable configuration indicated by the label $\left[l_{A}, l_{B}\right]$ changes.

\subsubsection{Effects of the axial flow}

The characteristics of the Kelvin modes strongly vary with the parameter $W_{0}$. Additional branch crossings involving smaller branch labels are obtained as $W_{0}$ increases. As explained in the previous section, resonances between $m_{A}=1$ and $m_{B}=2$, as well 

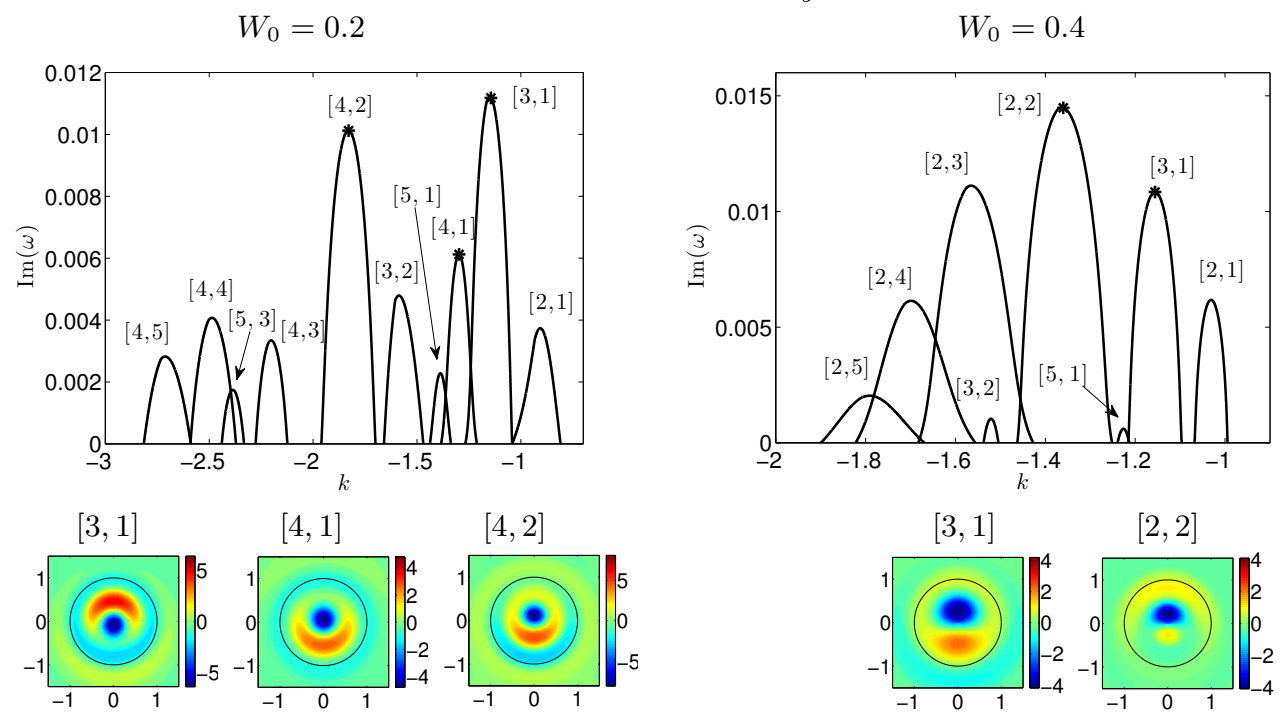

Figure 7. Top: Temporal growth rate of the curvature instability as a function of the axial wavenumber for the Batchelor vortex at $\varepsilon=0.2$ and $R e=5000$ for $W_{0}=0.2$ (left) and $W_{0}=0.4$ (right). Bottom: Vorticity contours in a $(x, y)$ cross section of modes $[3,1],[4,1]$, and $[4,2]$ for $W_{0}=0.2$ (left) and modes [3,1] and [2.2] for $W_{0}=0.4$ (right). See caption of Fig. 5 for more information.

as between $m_{A}=2$ and $m_{B}=3$ become a priori possible (see Fig. 2). However, they involve very high branch labels which implies that they never become the most unstable modes for moderate Reynolds numbers $\left(R e \leqslant 10^{5}\right)$.

For the parameters $W_{0}=0.1,0.2,0.3,0.4$ and 0.5 , we have considered the crossing points of the seven first branches of the Kelvin modes $m_{A}=0$ and $m_{B}=1$. Each crossing point corresponds to a mode resonance. At each crossing point, we have computed the coefficients of the growth rate expression. In Fig. 7, we have plotted the growth rate curves obtained from Eq. (3.14) for $W_{0}=0.2$ and 0.4, and for $\varepsilon=0.2$ and $R e=5000$. Contrarily to the Lamb-Oseen vortex, more resonant configurations can now become unstable. Moreover, they involve smaller branch labels. The spatial structure of the main resonant configurations have also been provided in Fig. 7 for this set of parameters. As in Fig. 5, we have plotted the vorticity contour for a particular phase which maximizes the relative amplitude of the Kelvin mode $m_{A}=0$. Note that the spatial structure of the resonant mode $[3,1]$ is different for $W_{0}=0.2$ and $W_{0}=0.4$ : this difference is not only associated with the different values of the coefficient $B / A$ obtained from (3.6), but also with an effect of $W_{0}$ on the Kelvin modes.

If we take the maximum value of the growth rate over all possible $k$ for each $\varepsilon$ and $R e$, we obtain the plots shown in Fig. 8. The same colormap and contour levels have been used as in Fig. 6 for comparison. We clearly see that the growth rates are larger in the presence of axial flow. The region of instability is also much larger. In these plots, we have indicated the labels of the most unstable modes. As for the Lamb-Oseen vortex, the most unstable configuration changes as $\varepsilon$ or $R e$ varies. However, the branch labels of the Kelvin modes are now smaller in the presence of axial flow. This property explains in part the larger growth rates of the configurations with jet. Indeed, the viscous damping of the modes with the smallest labels is the weakest. The impact of viscosity is therefore weaker on these modes. Yet, the resonant configuration with the smallest labels are 


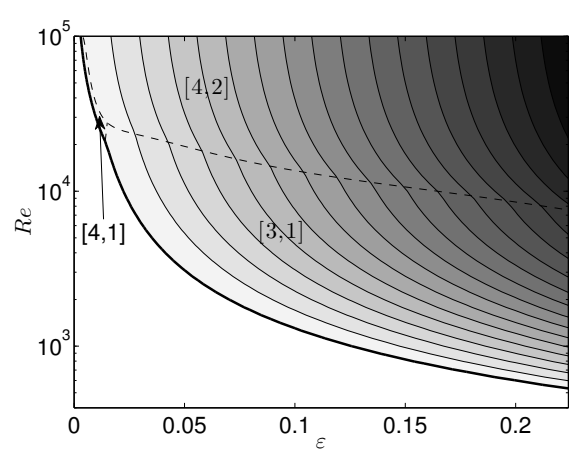

(a)

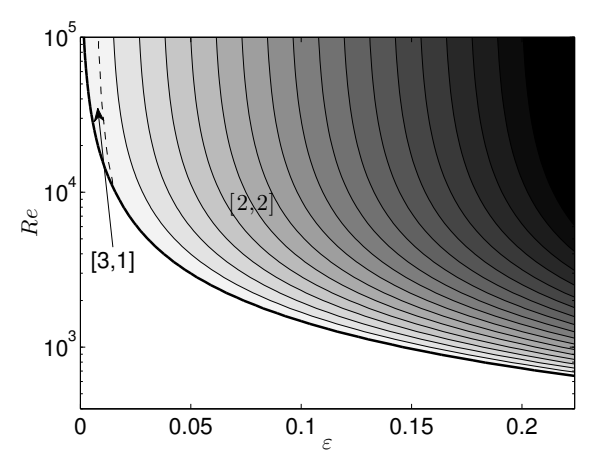

(b)

FiguRE 8. Maximum growth rate contours of the curvature instability in the $(\varepsilon, R e)$ plane for the Batchelor vortex. (a): $W_{0}=0.2 ;(\mathrm{b}): W_{0}=0.4$. See caption of Fig. 6 .

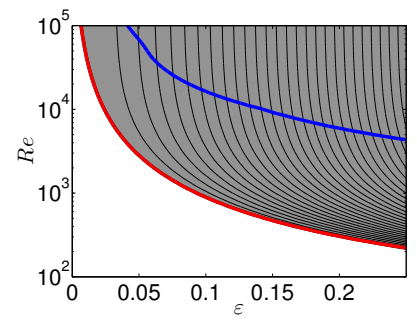

(a)

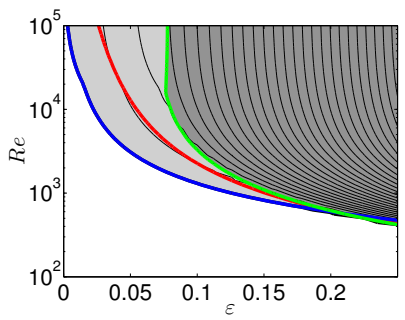

(b)

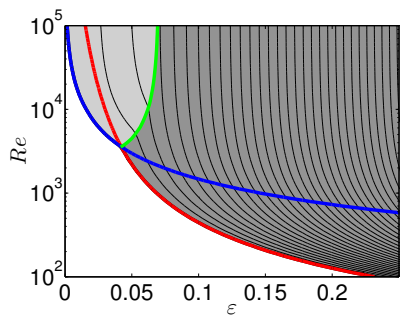

(c)

FIGURE 9. Maximum growth rate contours of short-wavelength instabilities (curvature instability and elliptic instability) in a vortex ring with a Batchelor vortex profile in the $(\varepsilon=a / R, R e)$ plane. Contours are every 0.002 . Blue line: marginal curve of the curvature instability $\left(\operatorname{Im}\left(\omega_{\text {curv }}\right)=0\right)$. Red line: marginal curve of the elliptic instability $\left(\operatorname{Im}\left(\omega_{\text {ellip }}\right)=0\right)$. Green line: Line of equal dominance of both instabilities $\left(\operatorname{Im}\left(\omega_{\text {ellip }}\right)=\operatorname{Im}\left(\omega_{\text {curv }}\right)\right)$. Elliptic instability dominates curvature instability in the dark grey region. (a) $W_{0}=0$; (b) $W_{0}=0.2$ (c) $W_{0}=0.4$.

not necessarily the most unstable because they may also exhibit a larger critical layer damping, or a smaller coupling coefficient $N$ [see equation (3.14)].

In tables 2 and 3 of appendix $\mathrm{C}$, we have provided the characteristics of the main resonant configurations for $W_{0}=0.2$ and $W_{0}=0.4$. The data for the other resonant configurations and for other values of $W_{0}$ are available as supplementary material.

\section{Competition with the elliptic instability in a vortex ring}

The results obtained in $\S 4$ can readily be applied to the vortex ring by using $\varepsilon=a / R$ where $R$ is the radius of the ring and $a$ the core radius.

As first shown by Widnall et al. (1974), the vortex ring is also subject to the elliptic instability. This instability appears at the order $\varepsilon^{2}$, so it is a priori smaller. Yet, the short wavelength instability observed experimentally in a vortex ring without swirl has always been attributed to the elliptic instability (see the review by Shariff \& Leonard 1992). It is therefore natural to provide a more precise comparison of the growth rates of both instabilities.

In BRLD16, we have obtained theoretical predictions for the elliptic instability in a vortex ring with a Batchelor profile. As for the curvature instability, growth rate contour plots can be obtained for the elliptic instability in a $(\varepsilon, R e)$ plane using the data of this paper. It should be noted that an error of a factor 2 was found in some of the coefficients 
of the elliptic instability growth rate formula. This error, which is corrected in appendix $\mathrm{D}$, does not affect the main conclusion of this paper but modifies the relative importance of the elliptic instability with respect to the curvature instability.

The comparison of the elliptic instability with the curvature instability is shown in Fig. 9 for three values of the axial flow parameters $\left(W_{0}=0,0.2,0.4\right)$. In this figure, we have plotted the largest value of both instability growth rates in the $(\varepsilon, R e)$ plane. We have also indicated where each instability appears and becomes dominant over the other one. Interestingly, we observe that depending on the value of $W_{0}$ the region of dominance of the curvature instability changes. For the case without axial flow [Fig. 9(a)], the elliptic instability domain is larger than the curvature instability domain and the elliptic instability is always the dominant instability. For the other two cases $W_{0}=0.2$ and $W_{0}=0.4$, the situation is different: there is a balance between both instabilities. For both cases, curvature instability is dominant over the elliptic instability for small $\varepsilon$ while it is the opposite for large $\varepsilon$. Yet, there are some differences between both cases. For $W_{0}=0.2$, we observe that the curvature instability is the first instability to appear as $R e$ is increased for all $\varepsilon<0.2$. For $W_{0}=0.4$, the elliptic instability domain is larger and extends to smaller values of the Reynolds numbers than for the other two cases. It is also the dominant instability for all Reynolds numbers as soon as $\varepsilon$ is larger than 0.1.

These plots have interesting implications. First, it explains why the curvature instability has never been observed in vortex ring without swirl. For such a vortex ring, the elliptic instability is always stronger than the curvature instability. Second, it implies that the curvature instability should be visible in a vortex ring with swirl if $\varepsilon$ is smaller than 0.1 and the Reynolds number larger than 10000 .

It should also be noted that due to the different inviscid scalings, which are in $\varepsilon$ for the curvature instability growth rate and in $\varepsilon^{2}$ for the elliptic instability growth rate, the curvature instability should always become dominant over the elliptic instability whatever $W_{0}$ if $\varepsilon$ is sufficiently small and the Reynolds number sufficiently large. This tendency is clearly seen in figures $9(\mathrm{~b}, \mathrm{c})$ for $W_{0}=0.2$ and $W_{0}=0.4$. For $W_{0}=0$ (fig.9(a)), the change of dominance of both instabilities occurs for a much larger Reynolds number.

\section{Conclusion}

In this work, we have provided the characteristics of the curvature instability for a Batchelor vortex for several axial flow parameters. We have shown that although a same resonant coupling is active as in a Rankine vortex, the characteristics of the resonant configurations are very different owing to the critical layer damping of many Kelvin modes. We have shown that this effect precludes the resonance of Kelvin modes with azimuthal wavenumbers larger than $m=3$. Moreover, when it occurs, the resonance of modes $\left(m_{A}, m_{B}\right)=(1,2)$ or $(2,3)$, involves a Kelvin mode with a very high complexity (large branch label) which is strongly sensitive to viscous effects. For moderate Reynolds numbers $\left(R e \leqslant 10^{5}\right.$ ), we have then found that the most unstable configuration always involves Kelvin modes of azimuthal wavenumbers $m_{A}=0$ and $m_{B}=1$. We have analysed the condition of resonance of the 7 first branches (9 for the Lamb-Oseen vortex) for several axial flow parameters to identify the most unstable configuration.

For the case without axial flow (Lamb-Oseen vortex), we have shown that the most unstable configuration involves the first branch of the Kelvin mode of azimuthal wavenumber $m_{B}=1$ and the seventh to nineth branch of the Kelvin mode of azimuthal wavenumber $m_{A}=0$, depending on the Reynolds number and $\varepsilon$ (for $R e \leqslant 10^{5}$ ). The high value of the branch label implies a larger viscous damping and therefore a weaker growth rate of the curvature instability for this case. In the presence of axial flow, resonant configura- 
tions with smaller branch labels were shown to become possible. The instability growth rate was then found to be larger than without axial flow. We have presented the characteristics of the most unstable configurations for two axial flow parameters $W_{0}=0.2$, $W_{0}=0.4$. The data provided as supplementary material can be used to obtain the instability characteristics for other values of $W_{0}\left(W_{0}=0,0.1,0.2,0.3,0.4,0.5\right)$.

We have applied our results to the vortex ring and analysed the competition of the curvature instability with the elliptic instability. We have shown that the elliptic instability is always dominant without axial flow. However, the situation changes in the presence of axial flow which provides hope in observing this instability in vortex rings with swirl.

The present results can also be applied to helical vortices as they only depend on the local vortex curvature. By contrast, the elliptic instability characteristics in helices depend on the helix pitch and on the number of helices (Blanco-Rodríguez \& Le Dizès 2016). Whether the curvature instability dominates the elliptic instability must then be analysed on a case by case basis. All the elements to perform such an analysis are now available.

Our analysis has been limited to a particular model of vortices. In the very large Reynolds number context of aeronautics, other models have been introduced to describe the vortices generated by wing tips (Moore \& Saffman 1973; Spalart 1998). It would be interesting to analyse the occurrence of the curvature instability in these models as well as the competition with the elliptic instability (Fabre \& Jacquin 2004a; Feys \& Maslowe 2016).

\section{Acknowledgments}

This work received support from the French Agence Nationale de la Recherche under the $\mathrm{A}^{*}$ MIDEX grant ANR-11-IDEX-0001-02, the LABEX MEC project ANR-11-LABX0092 and the ANR HELIX project ANR-12-BS09-0023-01.

\section{REFERENCES}

BAyly, B. J. 1986 Three-dimensional instability of elliptical flow. Phys. Rev. Lett. 57, 21602163.

Blanco-Rodríguez, F. J. \& Le Diż̀s, S. 2016 Elliptic instability of a curved Batchelor vortex. J. Fluid Mech. 804, 224-247.

Blanco-Rodríguez, F. J., Le Dizès, S., Selçuk, C., Delbende, I. \& Rossi, M. 2015 Internal structure of vortex rings and helical vortices. J. Fluid Mech. 785, 219-247.

CAllegari, A. J. \& Ting, L. 1978 Motion of a curved vortex filament with decaying vortical core and axial velocity. SIAM J. Appl. Math. 35, 148-175.

Crow, S. C. 1970 Stability theory for a pair of trailing vortices. AIAA J. 8 (12), 2172-2179.

Eloy, C. \& LE Diż̀s, S. 1999 Three-dimensional instability of Burgers and Lamb-Oseen vortices in a strain field. J. Fluid Mech. 378, 145-166.

Eloy, C. \& LE DizÈs, S. 2001 Stability of the Rankine vortex in a multipolar strain field. Phys. Fluids 13 (3), 660-676.

FABRE, D. \& JACQUin, L. 2004a Short-wave cooperative instabilities in representative aircraft vortices. Phys. Fluids 16, 1366-1378.

FABRE, D. \& JACQUin, L. $2004 b$ Viscous instabilities in trailing vortices at large swirl numbers. J. Fluid Mech. 500, 239-262.

Fabre, D., Sipp, D. \& Jacquin, L. 2006 The Kelvin waves and the singular modes of the Lamb-Oseen vortex. J. Fluid Mech. 551, 235-274.

Feys, J \& Maslowe, S. A. 2016 Elliptical instability of the Moore-Saffman model for a trailing wingtip vortex. J. Fluid Mech. 803, 556-590.

Fukumoto, Y. 2003 The three-dimensional instability of a strained vortex tube revisited. $J$. Fluid Mech. 493, 287-318. 
Fukumoto, Y. \& Hattori, Y. 2005 Curvature instability of a vortex ring. J. Fluid Mech. 526, $77-115$.

Hattori, Y. \& Fukumoto, Y. 2003 Short-wavelength stability analysis of thin vortex rings. Phys. Fluids 15, 3151-3163.

Hattori, Y. \& Fukumoto, Y. 2009 Short-wavelength stability analysis of a helical vortex tube. Phys. Fluids 21, 014104.

Hattori, Y. \& Fukumoto, Y. 2010 Short-wave stability of a helical vortex tube: the effect of torsion on the curvature instability. Theo. Comp. Fluid Dyn. 24, 363-368.

Hattori, Y. \& Fukumoto, Y. 2012 Effects of axial flow on the stability of a helical vortex tube. Phys. Fluids 24, 054102.

Hattori, Y. \& Fukumoto, Y. 2014 Modal stability analysis of a helical vortex tube with axial flow. J. Fluid Mech. 738, 222-249.

Lacaze, L., Ryan, K. \& Le Dizès, S. 2007 Elliptic instability in a strained Batchelor vortex. J. Fluid Mech. 577, 341-361.

LE DizÈs, S. 2004 Viscous critical-layer analysis of vortex normal modes. Stud. Appl. Math. 112 (4), 315-332.

LE Dizès, S. 2008 Inviscid waves on a Lamb-Oseen vortex in a rotating stratified fluid: consequences on the elliptic instability. J. Fluid Mech. 597, 283-303.

LE Dizès, S. \& FABRE, D. 2007 Large-Reynolds-number asymptotic analysis of viscous centre modes in vortices. J. Fluid Mech. 585, 153-180.

Le Dizès, S. \& LACAZE, L. 2005 An asymptotic description of vortex Kelvin modes. J. Fluid Mech. 542, 69-96.

Le Dizès, S. \& LAporte, F. 2002 Theoretical predictions for the elliptic instability in a twovortex flow. J. Fluid Mech. 471, 169-201.

Lifschitz, A. \& Hameiri, E. 1991 Local stability conditions in fluid dynamics. Phys. Fluids A 3 (11), 2644-2651.

Mayer, E. W. \& Powell, K. G. 1992 Viscous and inviscid instabilities of a trailing vortex. J. Fluid Mech. 245, 91-114.

Moore, D. W. \& Saffman, P. G. 1973 Axial flow in laminar trailing vortices. PRSA 333, 491-508.

Moore, D. W. \& SAfFman, P. G. 1975 The instability of a straight vortex filament in a strain field. Proc. R. Soc. Lond. A. 346, 413-425.

Quaranta, H. U., Bolnot, H. \& Leweke, T. 2015 Long-wave instability of a helical vortex. J. Fluid Mech. 780, 687-716.

Shariff, K. \& Leonard, A. 1992 Vortex rings. Annu. Rev. Fluid Mech. 24, 235-279.

SipP, D. \& JACQUIN, L. 2003 Widnall instabilities in vortex pairs. Phys. Fluids 15, 1861-1874.

Spalart, P. R. 1998 Airplane trailing vortices. Annu. Rev. Fluid Mech. 30, 107-138.

Tsai, C.-Y. \& WidnalL, S. E. 1976 The stability of short waves on a straight vortex filament in a weak externally imposed strain field. J. Fluid Mech. 73 (4), 721-733.

Whleffe, F. 1990 On the three-dimensional instability of strained vortices. Phys. Fluids A $2(1), 76-80$.

Widnall, S. E. 1972 The stability of a helical vortex filament. J. Fluid Mech. 54, 641-663.

Widnall, S. E., Bliss, D. \& Tsai, C.-Y. 1974 The instability of short waves on a vortex ring. J. Fluid Mech. 66 (1), 35-47.

Widnall, S. E. \& TsaI, C.-Y. 1977 The instability of the thin vortex ring of constant vorticity. Phil. Trans. R. Soc. London A 287, 273-305. 


\section{Appendix A. Dipolar correction}

The first order correction is given by

$$
\mathbf{U}_{1} \sim \varepsilon \operatorname{Re}\left(\mathbf{U}^{(1)} \mathrm{e}^{\mathrm{i} \varphi}\right)=\frac{\varepsilon}{2}\left(\begin{array}{l}
\mathrm{i} U^{(1)}(r) \\
V^{(1)}(r) \\
W^{(1)}(r) \\
P^{(1)}(r)
\end{array}\right) \mathrm{e}^{\mathrm{i} \varphi}+\text { c.c. },
$$

where

$$
\begin{aligned}
& U^{(1)}=\frac{\Psi^{(1)}}{r}, \\
& V^{(1)}=-\Psi_{r}^{(1)}+r V^{(0)}, \\
& W^{(1)}=-\frac{W_{r}^{(0)}}{V^{(0)}} \Psi^{(1)}+r W^{(0)}, \\
& P^{(1)}=-\zeta^{(0)} \Psi^{(1)}+V^{(0)} \Psi_{r}^{(1)}-r\left[V^{(0)}\right]^{2}-r\left[W^{(0)}\right]^{2},
\end{aligned}
$$

with

$$
\begin{aligned}
& \Psi^{(1)}(r)=V^{(0)}(r) \int_{0}^{r} \frac{\int_{0}^{z} V^{(0)}(\eta) F_{0}(\eta) \eta d \eta}{z\left[V^{(0)}(z)\right]^{2}} d z, \\
& F_{0}(\eta)=2 \eta \zeta^{(0)}(\eta)+V^{(0)}(\eta)+2 \eta \frac{W^{(0)}(\eta) W_{r}^{(0)}(\eta)}{V^{(0)}(\eta)} .
\end{aligned}
$$

We have used the index $r$ to denote derivative with respect to $r$ (for example $W_{r}^{(0)}=$ $\left.\partial_{r} W^{(0)}\right)$.

\section{Appendix B. Operators}

The operators appearing in equation (2.5) are given by

$$
\begin{gathered}
\mathbf{I}=\left[\begin{array}{llll}
1 & 0 & 0 & 0 \\
0 & 1 & 0 & 0 \\
0 & 0 & 1 & 0 \\
0 & 0 & 0 & 0
\end{array}\right], \quad \mathbf{P}=\left[\begin{array}{cccc}
W^{(0)} & 0 & 0 & 0 \\
0 & W^{(0)} & 0 & 0 \\
0 & 0 & W^{(0)} & 1 \\
0 & 0 & -1 & 0
\end{array}\right], \\
\mathbf{M}(-\mathrm{i} \partial \varphi)=\left[\begin{array}{cccc}
\Omega^{(0)} \mathrm{i} \partial_{\varphi} & -2 \Omega^{(0)} & 0 & \partial_{r} \\
-\zeta^{(0)} & \Omega^{(0)} \mathrm{i} \partial_{\varphi} & 0 & \frac{\mathrm{i}}{r} \partial_{\varphi} \\
-W_{r}^{(0)} & 0 & \Omega^{(0)} \mathrm{i} \partial_{\varphi} & 0 \\
\frac{1}{r}+\partial_{r} & -\frac{\mathrm{i}}{r} \partial_{\varphi} & 0 & 0
\end{array}\right], \\
\mathbf{V}\left(-\mathrm{i} \partial \varphi,-\mathrm{i} \partial_{s}\right)=\left[\begin{array}{cccc}
\Delta-\frac{1}{r^{2}} & \frac{2 \mathrm{i}}{r^{2}} \partial_{\varphi} & 0 & 0 \\
\frac{2 \mathrm{i}}{r^{2}} \partial_{\varphi} & \Delta-\frac{1}{r^{2}} & 0 & 0 \\
0 & 0 & \Delta & 0 \\
0 & 0 & 0 & 0
\end{array}\right],
\end{gathered}
$$




$\begin{array}{lrrrr}{\left[l_{A}, l_{B}\right]} & {[6,1]} & {[7,1]} & {[8,1]} & {[9,1]} \\ \omega_{c} & 0.134 & 0.124 & 0.115 & 0.108 \\ k_{c} & -1.247 & -1.346 & -1.426 & -1.493 \\ \operatorname{Im}\left(\omega_{B}\right) & -0.0059 & -0.0027 & -0.0011 & -0.0004 \\ Q_{A} & -0.102 & -0.088 & -0.078 & -0.069 \\ Q_{B} \times 10^{2} & 9.24-4.17 \mathrm{i} & 10.26-2.51 \mathrm{i} & 11.2-1.38 \mathrm{i} & 12-0.69 \mathrm{i} \\ V_{A} & -196.5 & -265.7 & -345.1 & -434.5 \\ V_{B} & -0.63+3.01 \mathrm{i} & -1.41+2.02 \mathrm{i} & -2.27+1.23 \mathrm{i} & -3.15+0.67 \mathrm{i} \\ C_{A B} \times 10^{3} & 14.4+0.9 \mathrm{i} & -10.9-0.5 \mathrm{i} & 8.2+0.2 \mathrm{i} & -6.3-0.1 \mathrm{i} \\ C_{B A} \times 10 & -1.26+0.47 \mathrm{i} & 1.51-0.27 \mathrm{i} & -1.69+0.14 \mathrm{i} & 1.82-0.06 \mathrm{i} \\ N \times 10^{2} & 4.35-0.64 \mathrm{i} & 4.07-0.27 \mathrm{i} & 3.74-0.10 \mathrm{i} & 3.39-0.03 \mathrm{i}\end{array}$

TABLE 1 . Characteristics of the first resonant configurations $\left(m_{A}, m_{B}\right)=(0,1)$ of label $\left[l_{A}, l_{B}\right]$ for the Lamb-Oseen vortex $\left(W_{0}=0\right)$.

\begin{tabular}{lrrr}
\hline$\left[l_{A}, l_{B}\right]$ & {$[3,1]$} & {$[4,1]$} & {$[4,2]$} \\
$\omega_{c}$ & 0.109 & 0.073 & 0.095 \\
$k_{c}$ & -1.144 & -1.298 & -1.829 \\
$\operatorname{Im}\left(\omega_{B}\right) \times 10^{3}$ & -0.75 & -0.02 & -0.004 \\
$Q_{A} \times 10^{2}$ & -7.94 & -4.66 & -3.83 \\
$Q_{B} \times 10^{2}$ & $20.68-1.67 \mathrm{i}$ & $26.21-0.01 \mathrm{i}$ & $20.12-0.14 \mathrm{i}$ \\
$V_{A}$ & -25.04 & -35.06 & -37.16 \\
$V_{B}$ & $-2.57+0.95 \mathrm{i}$ & $-5.90+0.01 \mathrm{i}$ & $-16.82+0.19 \mathrm{i}$ \\
$C_{A B} \times 10^{2}$ & $-2.64-0.039 \mathrm{i}$ & 1.11 & $-1.57-0.004 \mathrm{i}$ \\
$C_{B A} \times 10$ & $1.91-0.113 \mathrm{i}$ & -2.15 & $3.76-0.013 \mathrm{i}$ \\
$N \times 10^{2}$ & $7.09-0.16 \mathrm{i}$ & 4.90 & 7.70
\end{tabular}

TABLE 2. Same as table 1 for the Batchelor vortex with $W_{0}=0.2$.

$$
\mathbf{N}_{ \pm}^{(1)}\left(-\mathrm{i} \partial \varphi,-\mathrm{i} \partial_{s}\right)=\frac{1}{2}\left[\begin{array}{cccc}
D_{ \pm}^{(1)} \pm U_{r}^{(1)} & \frac{U^{(1)}}{r}+2 \frac{V^{(1)}}{r} & -2 W^{(0)} & 0 \\
V_{r}^{(1)}+\frac{V^{(1)}}{r} & D_{ \pm}^{(1)} \pm \frac{V^{(1)}}{r} \pm \frac{U^{(1)}}{r} & \pm 2 W^{(0)} & 0 \\
W_{r}^{(1)}-W^{(0)} & \pm \frac{W^{(1)}}{r} \mp W^{(0)} & D_{ \pm}^{(1)} \mp V^{(0)} & -r \mathrm{i} \partial_{s} \\
1 & \pm 1 & r \mathrm{i} \partial_{s} & 0
\end{array}\right]
$$

where

$$
\begin{aligned}
& D_{ \pm}^{(1)}= \pm U^{(1)} \partial_{r}-\frac{V^{(1)}}{r} \mathrm{i} \partial_{\varphi}-T_{w} \mathrm{i} \partial_{s}, \quad T_{w}=W^{(1)}+r W^{(0)} \\
& T_{v}=V^{(1)}+r V^{(0)}, \quad \Delta=\partial_{r}^{2}+\frac{1}{r} \partial_{r}+\frac{1}{r^{2}} \partial_{\varphi}^{2}+\partial_{s}^{2} .
\end{aligned}
$$

\section{Appendix C. Tables}

In this section, we provide the coefficients of the growth rate formula (3.14) for the dominant instability modes for the three cases $W_{0}=0, W_{0}=0.2$ and $W_{0}=0.4$. 


$\begin{array}{lrrr}{\left[l_{A}, l_{B}\right]} & {[2,1]} & {[3,1]} & {[2,2]} \\ \omega_{c} & 0.090 & 0.041 & 0.11 \\ k_{c} & -1.031 & -1.116 & -1.360 \\ \operatorname{Im}\left(\omega_{B}\right) \times 10^{3} & -0.086 & 0 & -0.382 \\ Q_{A} \times 10^{2} & -6.18 & -2.57 & -4.46 \\ Q_{B} \times 10^{2} & 35.08-0.45 \mathrm{i} & 42.01 & 31.03-1.45 \mathrm{i} \\ V_{A} & -8.31 & -13.38 & -9.13 \\ V_{B} & -4.50+0.21 \mathrm{i} & -7.94 & -16.98+1.25 \mathrm{i} \\ C_{A B} \times 10^{2} & 1.32+0.007 \mathrm{i} & -1.16 & -2.47-0.04 \mathrm{i} \\ C_{B A} \times 10 & -1.07+0.006 \mathrm{i} & 3.61 & 3.01-0.087 \mathrm{i} \\ N \times 10^{2} & 3.749 & 6.480 & 8.628-0.05 \mathrm{i}\end{array}$

TABLE 3. Same as table 1 for the Batchelor vortex with $W_{0}=0.4$.

\section{Appendix D. Elliptic instability of a curved Batchelor vortex - Corrigendum}

Due to a normalisation mistake, a systematic error has been made in the values of the coefficients $R_{A B}$ and $R_{B A}$ in the dispersion relation (4.7) of Blanco-Rodríguez \& Le Dizès (2016). The correct values are twice those indicated in this paper for all the modes. This modifies the values given in table 2 and formulas (C2n-q), (C3n-q), (C4n-q). For instance, in table 2 the correct value of $R_{A B}$ for the mode $(-2,0,1)$ at $W_{0}=0.4$ is $R_{A B}=2.302+0.382 \mathrm{i}$ instead of $R_{A B}=1.151+0.191 \mathrm{i}$.

This error affects the $y$-scale of the plots (c) and (d) of figure 5 which has to be multiplied by two, and those of figure 6 , which has to be divided by two. It also changes all the figures obtained in section 8 . The correct figures (available on request) are nevertheless qualitatively similar if we multiply the $y$-scale of all the plots by a factor 2 .

The comparison with Widnall \& Tsai (1977) done in section 8.1 for a vortex ring is also slightly modified. With the correct normalisation, the inviscid result of Widnall \& Tsai (1977) for the Rankine vortex is $\sigma_{\max } / \varepsilon^{2}=\left[(0.428 \log (8 / \varepsilon)-0.455)^{2}-0.113\right]^{1 / 2}$ while we obtain for the Lamb-Oseen vortex $\sigma_{\max } / \varepsilon^{2}=0.5171 \log (8 / \varepsilon)-0.9285$. The Lamb-Oseen vortex ring is thus less unstable than the Rankine vortex ring as soon as $\varepsilon>0.039$ for the same reason as previously indicated. 